\title{
The Phase of Oscillation in Cortical Network and Its Relationship with Firing Rate and Neuronal Activation
}

\author{
Daming WANG \\ Department of Computer Science and Technology \\ Tongji University \\ Shanghai, China \\ e-mail: 1e11damingwang@tongji.edu.cn
}

\author{
Yaoru SUN \\ Department of Computer Science and Technology \\ Tongji University \\ Shanghai, China \\ e-mail: yaoru@tongji.edu.cn
}

\begin{abstract}
Gamma-band neuronal oscillation and synchronization $(30-90 \mathrm{~Hz})$ are frequently observed in several cortical and subcortical circuits and are supposed to be involved in a variety of cognitive functions. The phase of the oscillation, referred to as one aspect of communication through coherence (CTC) hypothesis, often manifests itself as a substrate mechanism for feature coding, memory processing and behavior performing, and has quantitative relationships with input stimulus, neuronal activation and firing rate. In order to investigate oscillatory phase, we construct a simplified spiking neuronal network to simulate orientation columns in primary visual cortex. Input orientation, if preferred, can bring about higher firing rate and stronger neuronal activation for the corresponding column, which gives rise to smaller and more concentrated phases than those for other columns, with higher firing rate corresponding to smaller phase. Besides, time resolved spectral analyses with a sliding window are thoroughly elucidated.
\end{abstract}

Keywords-gamma-band; oscillation; phase; spike-LFP; orientation column; visual cortex; firing rate; leaky integrateand-fire

\section{INTRODUCTION}

Neuronal gamma-band oscillation and synchronization with the range of 30-90 $\mathrm{Hz}$ are abundant in numerous brain areas [1-3] and various species [4-6], which are proposed to underlie a diverse range of cognitive functions, including feature integration [7], signals routing [8], selective attention [9] and memory [10]. The role of gamma band oscillation for cognitive functions has been extensively investigated [11, 12] and a plausible mechanism is the hypothesis of communication through coherence (CTC) [8], the essence of which is that the rhythmic modulation of postsynaptic excitation engenders rhythmic modulations of synaptic input gain, and selective communication is implemented through selective coherence [13].

Two aspects of CTC concerning two signals are that if their amplitudes correlate (power correlation) or if their peaks and troughs align (phase coherence) [14]. For amplitude aspect, signal routing can be accurately achieved, because the spatial pattern of neuronal population firing rates can be reproduced in the spatial pattern of firing rate oscillation amplitude [15]. Selective attention to a sensory stimulus can be implemented through increasing the amplitude of the neuronal activity encoding that stimulus
[16]. Apart from amplitude aspect, the phase of oscillations, defined as the elapsing fraction relative to the beginning of the cycle, is hypothesized to allow information processing and transferring within and between brain circuits.

Oscillatory phase is suggested to be candidate mechanisms for several cognitive functions, for instance, feature coding [17] and information integration [7]. The electrophysiological experiment in visual cortex of awaken monkeys indicates that the phase can be coded systematically as a function of neuronal activation strength [18]. In the hippocampus, the phenomenon of theta-phase precession occurs and spatial information can also be encoded by theta phase [19]. Hippocampal theta phase is also assumed to encode and extract information in different phases of the theta cycle [3]. Other evidences with regard to the oscillatory phase are illustrated in phase-dependent coding of memorized objects [20] and phase dependent target detection [21].

It is indicated that the phase of oscillation has quantitative relationships with stimulus input, neuronal activation [18, 22]. The gamma cycle hypothesis proposes that the excitatory input can be transferred into a temporal code. Stronger inputs or more excited neurons will discharge earlier [22]. The intracranial physiological experiment in awaken monkey's visual cortex confirms the hypothesis above. The phase of an individual neuron shifts systematically as a function of neuronal activation strength, with stronger neuronal activation bringing about earlier spikes [18]. Several computational and theoretical simulations also derive similar results. As the driving current is increased, the spike phase varies systematically, with stronger driving current corresponding to earlier phase and stimulus strength can be encoded by spike phase [23]. Another simulation study demonstrates that the phase of firing decreases when the amplitude of sinusoidal input increases [24]. Besides, the relative phase between two neuronal groups can determine their interaction strength [4, 11, 25].

The phase representation measures can be classified into two categories. The first one is relative phase, inspired by the classical coherence approach for EEG and MEG, which determines the phase differences between two signals across different trials [26, 27]. The second one is point phase with respect to spike-LFP, which calculates the phase for each spike point associated with its background LFP oscillation 
[27].

In this paper, we explore the relationships among input stimulus, neuronal excitation, firing rate and spike-LFP phase. Network construction, computational simulation and spectral analysis are inspired by the experiment in visual cortex of awaken monkeys [18]. We intend to reproduce more simulations to supplement the scarce data and varying conditions of physiological experiment. When the input orientation is similar to or as same as the preferred orientation of a certain column, higher firing rate and

$$
C_{m} \frac{\mathrm{d} V}{\mathrm{~d} t}=-g_{L}\left(V-V_{\text {rest }}\right)+g_{A M P A}\left(V_{E}-V\right)+g_{G A B A}\left(V_{I}-V\right)+I_{b g}
$$

, where the parameter $C_{m}$ is a membrane capacitance, $g_{L}$ a membrane leak conductance, $V_{\text {rest }}$ the resting potential, and $I_{b g}$ a constant tonic background current. Besides, the parameters $V_{E}$ and $V_{I}$ are excitatory and inhibitory reversal potential respectively. The conductance complies with (2) and (3).

$$
\begin{aligned}
\frac{\mathrm{d} g_{A M P A}}{\mathrm{~d} t} & =-\frac{g_{A M P A}}{\tau_{A M P A}} \\
\frac{\mathrm{d} g_{G A B A}}{\mathrm{~d} t} & =-\frac{g_{G A B A}}{\tau_{G A B A}}
\end{aligned}
$$

, where $\tau_{A M P A}$ and $\tau_{G A B A}$ are excitatory and inhibitory decay time constants, and the default values for all the parameters can be referred to the TABLE I in the following section.

\section{B. Network Architecture}

As elucidated in Fig. 1A, the architecture of neuronal network includes two parts, the left of which is six groups of Poisson neurons, supplying external spike inputs, and the right is six columns, simulating orientation columns in the primary visual cortex. There are altogether 1200 Poisson neurons, 200 for each Poisson group. Each of the six orientation columns consists of 200 excitatory and 50 inhibitory neurons respectively. The ratio of excitatory neurons is $80 \%$, consistent with the experimental evidence [29]. Hence there are totally 1200 excitatory neurons and 300 inhibitory neurons for six columns.

The excitatory and inhibitory neurons in each of the six columns are assigned with a preferred orientation, $-\frac{\pi}{3}+\frac{\pi}{6} i$ for the $\mathrm{i}^{\text {th }}$ column, because of the orientation selectivity and bell-shape tuning curve of firing rate with respect to stimulus stronger neuronal activation will be brought about, which will in turn elicit smaller and more concentrated phase.

\section{METHODS}

\section{A. Neuronal Model}

All neurons in current paper are described as leaky integrate-and-fire neuronal model, the dynamics of which evolves according to the following equation [28]:

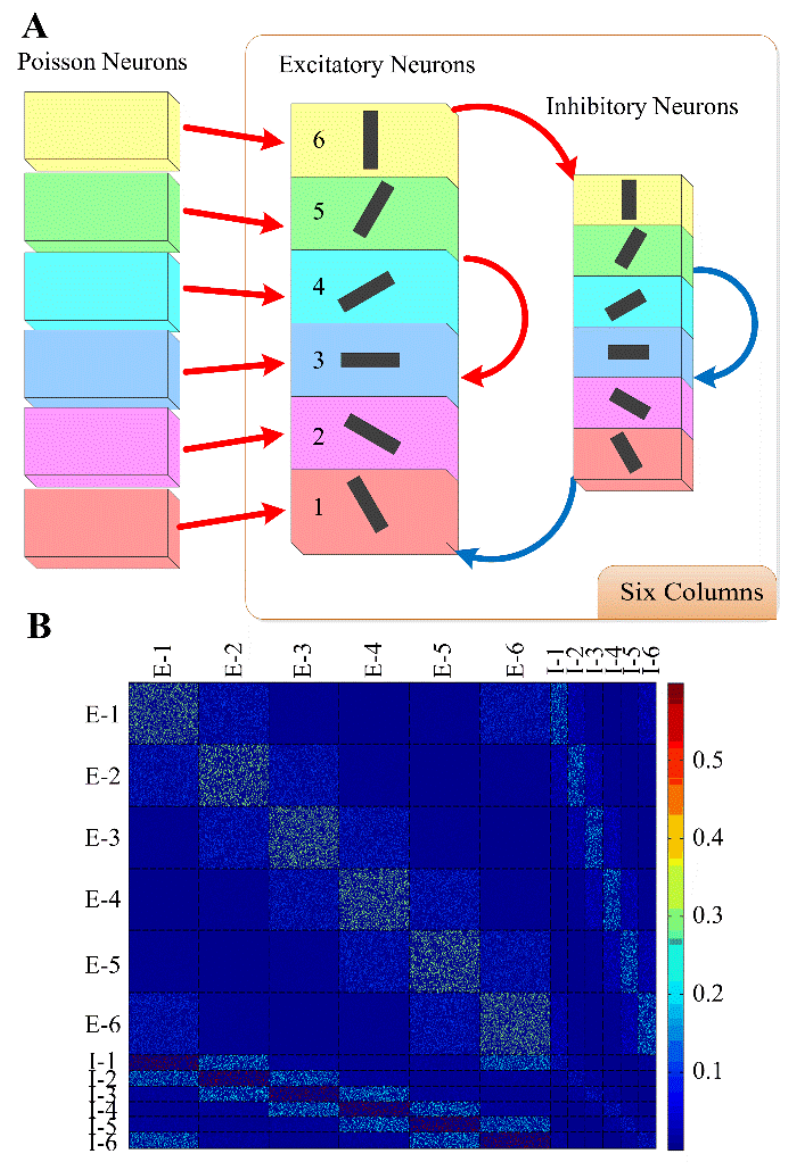

Figure 1. Network architecture and connection weight

orientation in the visual cortex [30]. Therefore the vector of preferred orientation is $\left\{-\frac{\pi}{3},-\frac{\pi}{6}, 0, \frac{\pi}{6}, \frac{\pi}{3}, \frac{\pi}{2}\right\}$. In addition, the neurons in the six Poisson groups are also designated with the same orientation preference as their corresponding orientation columns, the Poisson rate of which is determined through (4).

$$
R_{\text {input }}=\left[\cos \left(2\left(\theta_{\text {pref }}-\theta_{\text {stim }}\right)\right)+1\right] F_{\text {max }}
$$

, where $\theta_{\text {pref }}$ and $\theta_{\text {stim }}$ are the preferred orientation and the 
stimulus orientation respectively. Besides, $F_{\max }$ is maximal firing rate.

The connections between Poisson groups and orientation columns are forward and unidirectional, all having the same and constant weight $W_{f}$, with each Poisson group only projecting to its corresponding orientation column. The neurons within six orientation columns are mutually and reciprocally interconnected, both for excitatory and inhibitory neurons, which include connections from excitatory to excitatory neurons, excitatory to inhibitory, inhibitory to excitatory and inhibitory to inhibitory. The connection weight within six orientation columns obeys (5).

$$
W_{i j}=\operatorname{Wexp}\left(\beta\left[\cos \left(2\left(\theta_{\text {pre }}-\theta_{\text {post }}\right)\right)-1\right]\right)
$$

, where $\theta_{\text {pre }}$ and $\theta_{\text {post }}$ refers to as the preferred orientations for presynaptic and postsynaptic neurons respectively. The terms E-1 and I-1 in Fig. 1B denote excitatory and inhibitory neurons in the first column. Consequently, the connections within the same column can obtain larger connection weights, which gradually decrease across columns because of feature differences. In addition, the red and blue arrows represent excitatory and inhibitory synaptic connections, and all connections are established with the probability of $\varepsilon=0.2$.

The LFP data is established as the sum of the absolute values of excitatory, inhibitory synaptic and background currents from synapses of all pyramidal neurons within an adjacent group [31, 32].

$$
\mathrm{LFP}=\mathrm{R}\left(\sum_{i=1}^{n}\left(\left|I_{A M P A}\right|+\left|I_{G A B A}\right|+\left|I_{b g}\right|\right)\right)
$$

where the parameter $\mathrm{R}$ simulates the impedance of electrodes, which is utilized for recording the extracellular activities.

\section{Experiment Simulating}

We sequentially select 10 excitatory nearby neurons in each orientation column and record their spike activities and synapse currents, from which one LFP channel data is obtained. Consequently, six LFP and 60 neuronal channels are documented totally. We carry out 15 trials in the current experiment. For each trial, there is a pre-stimulus baseline period of $500 \mathrm{~ms}$, during which only the Poisson spike trains with a lower rate $F_{b g}$ and the constant background current $I_{b g}$ are delivered to the neurons in the orientation columns, in order to simulate the spontaneous activity of cerebral cortex. Then a constant orientation of 30 degrees, adopted to mimic drifting gratings, is presented for duration of $1500 \mathrm{~ms}$, which is the preferred orientation of the fourth orientation column.

For each spike from a certain neuronal channel, six LFP segments except the one within the same column are cut out and all LFP segments are decomposed into spike-triggered LFP spectrum through (7) [18].

$$
\bar{X}_{i}(f)=\frac{1}{5} \sum_{j=1}^{5} \frac{X_{i}^{j}(f)}{\left|X_{i}^{j}(f)\right|}
$$

, where the term $X_{i}^{j}(f)$ is the spike-triggered LFP spectrum from the $\mathrm{j}^{\text {th }}$ LFP channel and the $\mathrm{i}^{\text {th }}$ spike of a certain neuronal channel. Then we can easily derive the spike-LFP phase by means of (8) [18].

$$
\Theta_{i}=\operatorname{angle}\left(\bar{X}_{i}(f)\right)
$$

, the angle function derives phase angle of the input spectrum. Besides, the oscillatory phase used in our experiment is the mean spike-LFP phase across trials.

TABLE I. PARAMETER SETTINGS

\begin{tabular}{|c|c|c|c|}
\hline Parameter & Value & Parameter & Value \\
\hline$C_{m}$ & $250 \mathrm{pF}$ & $g_{L}$ & $10 \mathrm{nS}$ \\
\hline$V_{\text {rest }}$ & $-65 \mathrm{mV}$ & $V_{\text {thres }}$ & $-45 \mathrm{mV}$ \\
\hline$I_{b g}$ & $300 \mathrm{pA}$ & $V_{E}$ & $0 \mathrm{mV}$ \\
\hline$V_{I}$ & $-75 \mathrm{mV}$ & $\tau_{A M P A}$ & $5 \mathrm{~ms}$ \\
\hline$\tau_{G A B A}$ & $10 \mathrm{~ms}$ & $T_{\text {ref }}$ & $5 \mathrm{~ms}$ \\
\hline$F_{\max }$ & $40 \mathrm{~Hz}$ & $\varepsilon$ & 0.2 \\
\hline$\beta$ & 2 & $W_{f}$ & 0.05 \\
\hline $\mathrm{R}$ & $1.0 \mathrm{M} \Omega$ & $F_{b g}$ & $3 \mathrm{~Hz}$ \\
\hline
\end{tabular}

Ultimately, there are time resolved analyses with a sliding window illustrated, with the window length and time step being $75 \mathrm{~ms}$ and $10 \mathrm{~ms}$ respectively. All the simulations of neuronal model and network construction are implemented based on an open source Python package of Brian simulator [33]. The following spectral analyses are executed on the basis of the FieldTrip MATLAB toolbox [34]. The time step for numerical integration is $0.1 \mathrm{~ms}$. The parameters are all set according to the TABLE I.

\section{RESULTS}

\section{A. Neuronal Activity, LFP and Firing Rate}

The spike raster of $200 \mathrm{~ms}$ for Poisson, excitatory and inhibitory neurons are thoroughly demonstrated in the Fig. 2A. Each interval of vertical coordinate represents one orientation column. The outputs of Poisson neurons, supplemented by a constant background current, are delivered to the column network, and vector of Poisson rate 
A1

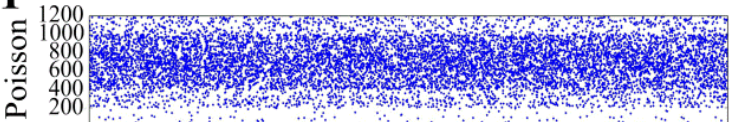

A2

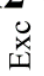

I

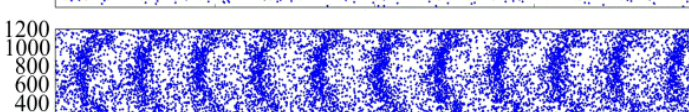

A3
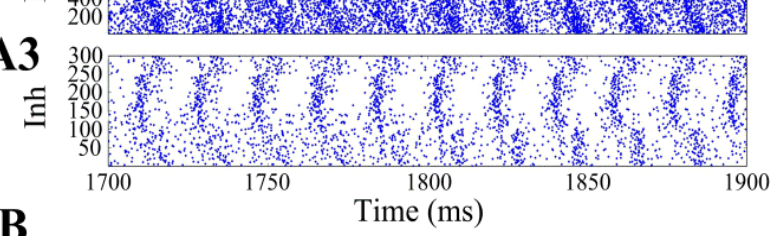

B

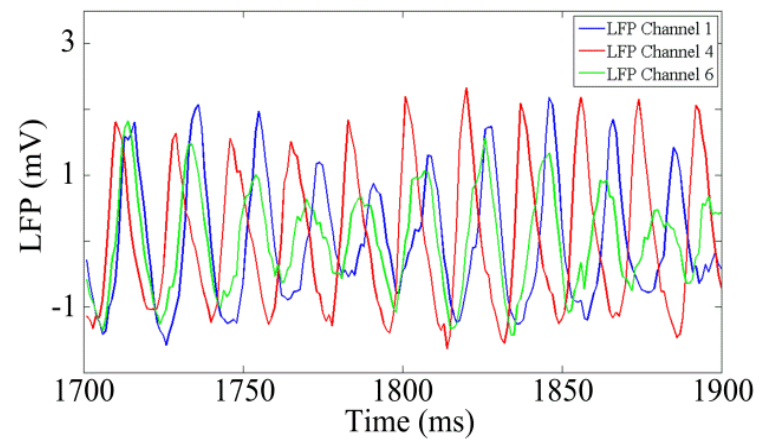

Figure 2. Spike raster and LFP. (A) Spike raster of $200 \mathrm{~ms}$ period for (A1) Poisson neurons, (A2) Excitatory neurons, (A3) Inhibitory neurons.

(B) LFP time sequences for the first, fourth and sixth channels.

is $\{3 \mathrm{~Hz}, 23 \mathrm{~Hz}, 63 \mathrm{~Hz}, 83 \mathrm{~Hz}, 63 \mathrm{~Hz}, 23 \mathrm{~Hz}$ (Fig. 3A). The spike raster of Poisson neurons is densest in the fourth group and gradually decreases upwards and downwards to other columns (Fig. 2A1). Consistent with vivo and vitro evidences [9], individual neurons fire irregularly and sparsely, nevertheless the population of both excitatory and inhibitory neurons fluctuate approximately at a certain frequency. Strong oscillation, at a frequency of $55 \mathrm{~Hz}$, emerges in both principal and inhibitory neurons during the period of $200 \mathrm{~ms}$ (Fig. 2A2 and 2A3). Further spectral analyses, i.e. spike-LFP phase, are all restricted to this frequency band.

Three LFP oscillatory traces are recorded from the first, fourth and sixth orientation columns, the peaks of which are totally different (Fig. 2B). Spikes discharge with higher probability to the troughs of the LFP oscillation [32], neurons in the fourth column are more likely to fire early, and then the sixth and first column, which is the reason why different oscillatory phases generated.
A

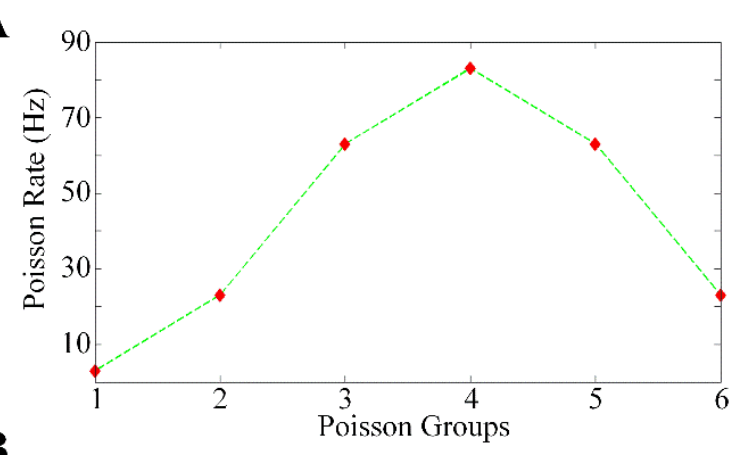

B

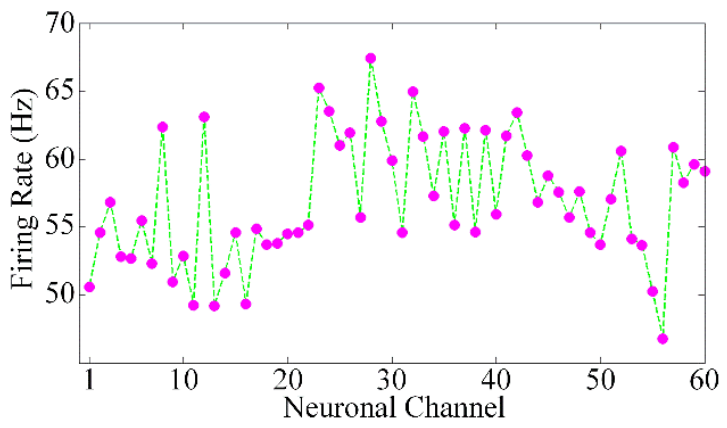

Figure 3. Neuronal firing rates. (A) Poisson rates for six Poisson groups.

(B) Neuronal firing rates for 60 neuronal channels across 15 trials.

Furthermore, we compute the firing rates of 60 neuronal channels across 15 trials. Though to a certain extent do the firing rates of all neuronal channels fluctuate, neurons from the third to fifth columns have higher firing rates, the ones from other columns with lower firing rates (Fig. 3B).

\section{B. Oscillatory Phase and Its Relationship with Firing Rate}

For each orientation column, 10 oscillatory phases, derived from 10 neuronal channels, are depicted in polar coordinates. The radial length equals to unit length, because the amplitude is normalized and not taken into account. The oscillatory phases for the third, fourth and fifth columns are more concentrated, and the ones for other columns are 

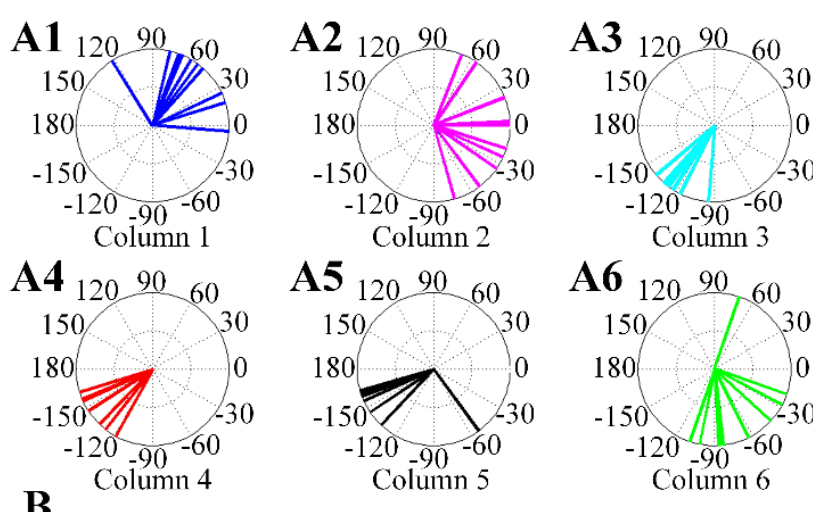

B
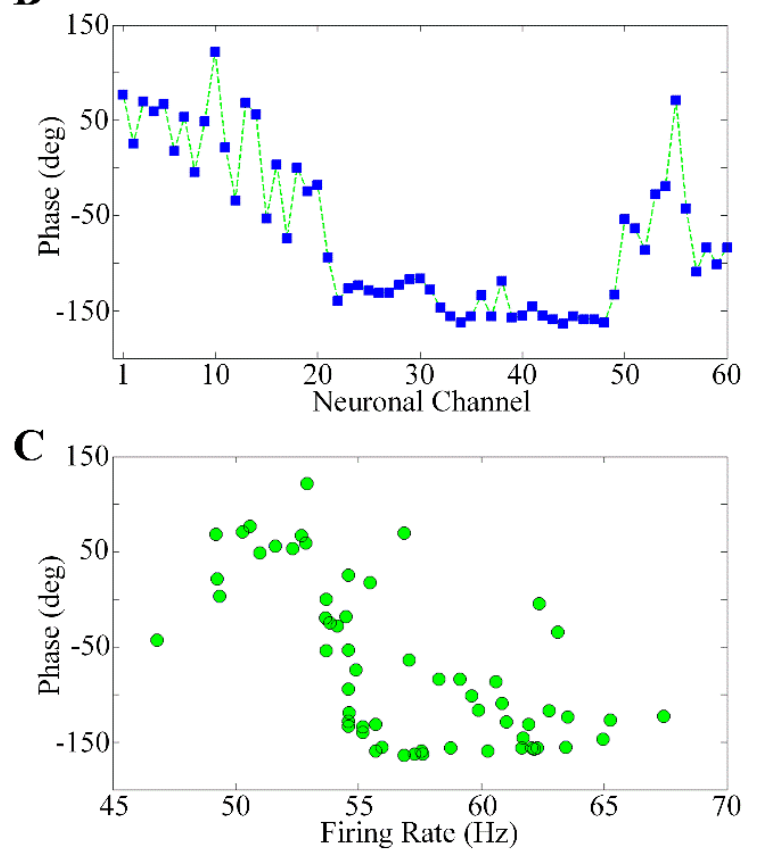

Figure 4. Oscillatory phases. (A) Polar plot of oscillatory phases for six columns. (B) Phases of neuronal channels in Cartesian coordinates. (C) Oscillatory phase as a function of firing rate.

considerably scattered. Besides, six mean phase centers are dramatically different (Fig. 4A). As indicated in Fig. 4B, the oscillatory phases of all neuronal channels fluctuate significantly, with the phases from the third to fifth columns smaller than the ones from other columns.

Because the input orientation of 30 degrees is similar to the preferred orientations from the third to the fifth columns, which brings about stronger Poisson inputs, and then higher firing rates and stronger neuronal activation. Thus the spikes from these columns are more likely to fire earlier and be focused on a certain part of LFP oscillations. Then smaller gamma phase and lower phase dispersions are generated [18]. Moreover, different mean phase centers arise from diverse firing rates and neuronal activations for six orientation columns.

The oscillatory phases are inversely proportional to the neuronal firing rates, with higher firing rate corresponding to smaller phase (Fig. 4C), which is in accordance with the hypothesis of gamma cycle [22] and the monkey experiment
[18].

\section{Time Resolved Analysis}

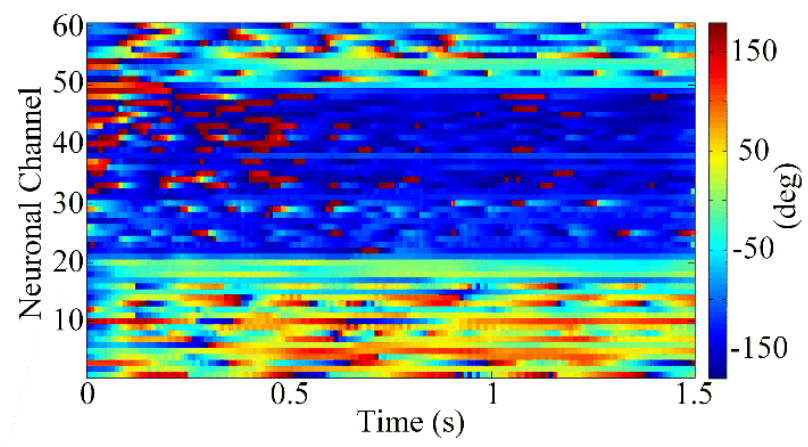

Figure 5. Oscillatory phases for neuronal channels across time with a sliding window

During the time resolved analysis with a sliding window (Fig. 5), the distribution of oscillatory phases across neuronal channel for any time point is consistent with the result in Fig. 4B. The phases for the third, fourth and fifth columns are smaller than those for other columns. Besides, the phases for the first, second and sixth columns fluctuate more dramatically across time axis than those for the other columns. In addition, there exists a stimulus onset transient effect imposed on the beginning period, during which phases from the third to fifth columns vary significantly.

\section{CONCLUSIONS}

In order to adequately investigate the oscillatory phase and its role in cognitive functions, we construct a neuronal network with six Poisson groups, delivering external inputs, and six columns, simulating orientation columns in primary visual cortex. The network constructing, data recording and spectral analyzing are to some extent analogous to the physiological experiment of awaken monkeys [18]. We choose the leaky integrate-and-fire neuronal model because of its computational efficiency and biological plausibility, which however can be substituted by the Hodgkin-Huxley model. If the preferred orientation of one column is close or equivalent to the input orientation stimulus, the neurons within this column will obtain higher firing rate and stronger activation, thereby obtaining earlier spike and smaller oscillatory phase. Besides, the phases are more concentrated.

The investigation in our experiment confirms the gamma cycle hypothesis [22] and the physiological experiment in visual cortex of awaken monkeys [18]. The gamma cycle hypothesis suggests that the amplitude of excitatory drive is coded into phase values which quantify how much a spike precedes the peak of a gamma cycle, and stronger inputs or more excited neurons will fire earlier in the gamma cycle [22]. The monkey experiment reveals that the spike times of individual neurons varies according to neuronal activation, and stronger neuronal activation gives rise to spikes earlier in the gamma cycle [18]. It is suggested in our experiment that oscillatory phase can be coded as a function of neuronal activation, with smaller phase corresponding to stronger inputs, greater neuronal activation and larger firing rate. 
Several computational studies obtain similar consequences, but the phases utilized in their experiments are all in time domain. In Tiesinga's experiment [23], the synchronous activities are modeled as a periodic sequence of Gaussian peaks, whose period is set as a constant time, and the phases are also represented as time. In another experiment [24], the inputs are directly modeled as sinusoidal signals, and the phases are evaluated with respect to the sinusoidal inputs. However, the phases adopted in our experiment are obtained from spike-LFP spectrum, which is within frequency domain.

\section{ACKNOWLEDGEMENTS}

This work was supported by the National Natural Science Foundation of China (61173116). We thank Haibo Shi in the laboratory for the helpful discussion and valuable advice.

\section{REFERENCES}

[1] Gray, C.M., et al., Oscillatory responses in cat visual cortex exhibit inter-columnar synchronization which reflects global stimulus properties. Nature, 1989. 338(6213): p. 334-7.

[2] Spellman, T., et al., Hippocampal-prefrontal input supports spatial encoding in working memory. Nature, 2015. 522(7556): p. 309-+.

[3] Igarashi, K.M., et al., Coordination of entorhinal-hippocampal ensemble activity during associative learning. Nature, 2014. 510(7503): p. 143-+.

[4] Womelsdorf, T., et al., Modulation of neuronal interactions through neuronal synchronization. Science, 2007. 316(5831): p. 1609-1612.

[5] Saalmann, Y.B., et al., The pulvinar regulates information transmission between cortical areas based on attention demands. Science, 2012. 337(6095): p. 753-6.

[6] Canolty, R.T., et al., High gamma power is phase-locked to theta oscillations in human neocortex. Science, 2006. 313(5793): p. 16261628.

[7] Brunet, N., et al., Visual cortical gamma-band activity during free viewing of natural images. Cereb Cortex, 2015. 25(4): p. 918-26.

[8] Fries, P., A mechanism for cognitive dynamics: neuronal communication through neuronal coherence. Trends in Cognitive Sciences, 2005. 9(10): p. 474-480.

[9] Fries, P., et al., Modulation of oscillatory neuronal synchronization by selective visual attention. Science, 2001. 291(5508): p. 1560-1563.

[10] Fell, J. and N. Axmacher, The role of phase synchronization in memory processes. Nature Reviews Neuroscience, 2011. 12(2): p. 105-U1500.

[11] Fries, P., Neuronal gamma-band synchronization as a fundamental process in cortical computation. Annual Review of Neuroscience, 2009. 32(1): p. 209-224.

[12] Buzsaki, G. and X.J. Wang, "Mechanisms of Gamma Oscillations." Annual Review of Neuroscience, 2012. 35(1): p. 203-225.

[13] Fries, P., Rhythms for Cognition: Communication through Coherence. Neuron, 2015. 88(1): p. 220-235.

[14] Harris, A.Z. and J.A. Gordon, Long-range neural synchrony in behavior. Annual Review of Neuroscience, 2015. 38(1): p. 171-94.

[15] Akam, T. and D.M. Kullmann, Oscillations and filtering networks support flexible routing of information. Neuron, 2010. 67(2): p. 308-
20.

[16] Zeitler, M., P. Fries and S. Gielen, Biased competition through variations in amplitude of gamma-oscillations. Journal of Computational Neuroscience, 2008. 25(1): p. 89-107.

[17] Kayser, C., et al., Spike-phase coding boosts and stabilizes information carried by spatial and temporal spike patterns. Neuron, 2009. 61(4): p. 597-608.

[18] Vinck, M., et al., Gamma-phase shifting in awake monkey visual cortex. Journal of Neuroscience, 2010. 30(4): p. 1250-1257.

[19] O'Keefe, J. and M.L. Recce, Phase relationship between hippocampal place units and the EEG theta rhythm. Hippocampus, 1993. 3(3): p. 317-30.

[20] Siegel, M., M.R. Warden and E.K. Miller, Phase-dependent neuronal coding of objects in short-term memory. Proceedings of the National Academy of Sciences of the United States of America, 2009. 106(50): p. 21341-21346.

[21] Helfrich, R.F., et al., Entrainment of brain oscillations by transcranial alternating current stimulation. Current Biology, 2014. 24(3): p. 333-

[22] Fries, P., D. Nikolic and W. Singer, The gamma cycle. Trends in neurosciences, 2007. 30(7): p. 309-316.

[23] Tiesinga, P.H. and T.J. Sejnowski, Mechanisms for phase shifting in cortical networks and their role in communication through coherence. Frontiers in Human Neuroscience, 2010. 4(196).

[24] Gielen, S., M. Krupa and M. Zeitler, Gamma oscillations as a mechanism for selective information transmission. Biological Cybernetics, 2010. 103(2): p. 151-165.

[25] Buehlmann, A. and G. Deco, Optimal information transfer in the cortex through synchronization. PLoS Computational Biology, 2010. 6(e10009349): p. e1000934.

[26] Vinck, M., et al., The pairwise phase consistency: A bias-free measure of rhythmic neuronal synchronization. NeuroImage, 2010. 51(1): p. 112-122.

[27] Vinck, M., et al., Improved measures of phase-coupling between spikes and the Local Field Potential. Journal of Computational Neuroscience, 2012. 33(1): p. 53-75.

[28] Vogels, T.P., et al., Inhibitory Plasticity Balances Excitation and Inhibition in Sensory Pathways and Memory Networks. Science, 2011. 334(6062): p. 1569-1573.

[29] Hu, H., J. Gan and P. Jonas, Fast-spiking, parvalbumin $(+)$ GABAergic interneurons: From cellular design to microcircuit function. Science, 2014. 345(12552636196): p. 529-+.

[30] Ferster, D. and K.D. Miller, Neural mechanisms of orientation selectivity in the visual cortex. Annual review of neuroscience, 2000. 23(1): p. 441-471.

[31] Mazzoni, A., et al., Encoding of naturalistic stimuli by local field potential spectra in networks of excitatory and inhibitory neurons. PLoS Computational Biology, 2008. 4(12): p. e1000239.

[32] Barardi, A., B. Sancristobal and J. Garcia-Ojalvo, Phase-coherence transitions and communication in the gamma range between delaycoupled neuronal populations. PLoS Computational Biology, 2014. 10(7): p. e1003723.

[33] Goodman, D. and R. Brette, Brian: a simulator for spiking neura networks in Python. Frontiers in neuroinformatics, 2008. 2.

[34] Oostenveld, R., et al., FieldTrip: open source software for advanced analysis of MEG, EEG, and invasive electrophysiological data. Computational intelligence and neuroscience, 2010. 2011. 